\title{
Novelty and significance as determiners of the GSR index of the orienting reflex
}

\author{
IRVING MALTZMAN and BARRY LANGDON \\ University of California, Los Angeles, California
}

\begin{abstract}
Fifty-six students in each of two groups heard an innocuous tone of constant interstimulus interval (ISI) repeatedly presented. Half the students were instructed to sit quietly and listen, whereas for half the students the tone was a signal in a reaction time experiment. The GSR was recorded continuously throughout the experiment. Following training with a constant ISI, the tone was presented in a temporal generalization test series with shorter and longer ISIs. Response speed manifested a symmetrical gradient of generalization on all generalization test series. The greater the change in ISI, the slower the response speed. On the first temporal generalization test, the GSR induced by the signal stimuli showed a symmetrical gradient, whereas generalization to nonsignificant stimuli was asymmetrical, with only longer ISIs showing increased GSR responsivity. Temporal generalization to the significant stimuli also developed an asymmetry after repeated training and generalization test series. These results suggest that the effects of novelty are at least in part dependent for their appearance upon the significance of the stimuli involved.
\end{abstract}

A growing, salutary interest in research and theory concerning the complex determiners of measures of the orienting reflex (OR), beyond those of simple novelty or stimulus change, has recently developed (e.g., Bernstein, 1979; Maltzman, 1979a, 1979b; O'Gorman, 1979; Siddle, 1979). Stimulus significance is one of these complex determiners. It is particularly evident when established by instructions that impart signal value to stimuli, that is, instructions to perform an overt (Luria \& Vinogradova, 1959; Maltzman \& Raskin, 1965, 1979) or covert act on signal (Maltzman, Gould, Pendery, \& Wolff, 1977; Maltzman \& Raskin, 1965, 1979). Aside from occasional failures (e.g., Barry, 1977; Becker \& Shapiro, 1980), which may be due to complexity of instructions and to difficulty in specifying the essential variables contained in instructions, as well as a variety of other methodological problems, the great majority of experiments report that signal value does enhance the psychophysiological changes reflecting an OR (e.g., Latash, 1968; Luria, 1973; Luria \& Homskaya, 1970; Luria \& Vinogradova, 1959; Maltzman, Gould, Barnett, Raskin, \& Wolff, 1979; Maltzman \& Raskin, 1979; Sokolov, 1963).

One explanation for the enhancement of ORs as a consequence of the signal value of a stimulus is that

This study was made possible in part by funds provided by U.S. Public Health Service Research Grant MH 04634 and by the Carnegie Corporation of New York. Requests for reprints should be sent to Irving Maltzman, Department of Psychology, University of California, Los Angeles, California 90024.

-Article accepted by previous editor, Richard F. Thompson the performance requirement increases the overall level of arousal. Heightened GSRs to the signal stimuli therefore occur as a consequence of this nonspecific increase in arousal (O'Gorman, 1979). The effects of increased signal value on augmented GSRs reflecting heightened orienting may be produced by arousal under some circumstances, as proposed by O'Gorman (1979), but it is clear that heightened arousal cannot account for augmented ORs induced by significant stimuli under all conditions. Our first experiment on this problem was explicitly designed to examine, as well as control for, nonspecific effects such as arousal (Maltzman \& Raskin, 1965). Significant increases in selective or differential orienting to one word among many were obtained as a result of having it serve as a cue for overt or covert responses. Such increases in selective ORs cannot be explained in terms of increases in nonspecific factors such as arousal or drive (Maltzman, 1971; Maltzman \& Raskin, 1979).

Maltzman (1979a, 1979b) has argued that whereas novelty is an important determiner of the OR, its effectiveness in turn is dependent upon the state of the organism at the moment. Momentary sets, motives, and longer standing dispositions, attitudes, etc., may predetermine the noteworthiness or significance of stimuli such that a stimulus change in one environmental setting or state of the individual may give rise to ORs, whereas that same kind of novelty will fail to yield such effects under different predisposing conditions. Translation of the problem of the relationship between the significance of stimuli and novelty into manageable laboratory problems is not always easy, but it is essential if the theory of the OR is to 
progress. One problem encountered in attempts to study the complex determiners of the $O R$ is the difficulty in independently manipulating novelty and the signal value of stimuli. Such independent manipulation is necessary in order to determine their effects separately and in combination. The present experiment was an attempt to explore the latter problem, the examination of the effects of novelty and of stimulus significance, by employing a temporal generalization paradigm.

The specific experimental problem addressed was whether changes in the temporal interval between innocuous tones have different effects upon the GSR index of the OR when the tones have signal value and when they do not. The procedure employed was one utilized in behavioral studies of temporal generalization (Desiderato, 1964). In this procedure, a stimulus is presented at a constant interval and, after repeated presentations at that interval, the duration of the interstimulus interval (ISI) is either decreased or increased. Response latency or speed has been the usual dependent variable in this situation. During training, the response speed increases. With changes in the ISI, lengthening or shortening, there may be a decrease in speed, the size of which tends to vary with the amount of change.

According to Sokolov (1963), any kind of change may evoke an OR, including changes in the temporal pattern of stimulation. A reasonable assumption, therefore, is that the introduction of generalization trials, changing the duration of the ISI, following training with a constant ISI would evoke an OR, and the greater the change in ISI, the greater the magnitude of the OR induced. A common observation has been that the evocation of an OR or the presentation of an attention-getting stimulus results in the temporary arrest of ongoing activity (Berlyne, 1960; Pillsbury, 1908; Sokolov, 1963). The implication is that the temporal generalization gradient, decreased speed of responding as a function of the amount of change in ISI, is inversely related to the magnitude of the OR evoked by the change in interval.

Our procedure for producing temporal generalization followed the one employed by Desiderato (1964) in his innocuous stimulus condition. We added a control group that received the same tone stimulus as the experimental group but did not overtly respond to it. Control students receiving nonsignificant stimuli should show less responsivity to the tones than experimental students for whom the stimulus was a signal to perform an overt response. Of greater theoretical interest are the electrodermal changes that might be induced during the generalization test series. We wished to determine whether or not changing the ISI between nonsignificant stimuli and between significant stimuli induces similar gradients of generalization of habituation of the GSR. According to our interpretation of Sokolov's (1963) neuronal model theory, ORs, as reflected by heightened GSRs, should occur as a function of the magnitude of the change in ISI from the interval present during training. Similar bidirectional changes should occur as a result of changed intervals both between significant stimuli and between nonsignificant stimuli, although the magnitude of the response should be greater in the former than the latter condition. If, however, the OR induced by novelty is in part a function of the state of the individual at the moment, including cortical sets or dominant foci (Maltzman, 1979a, 1979b), then the generalization gradients induced by novelty involving significant stimuli should differ from those involving nonsignificant stimuli. Precisely how generalization gradients induced by novelty should differ under different states cannot be predicted in our present state of ignorance.

It must be noted that the present procedure for investigating temporal generalization differs fundamentally from another kind of study that has been labeled temporal generalization and has been used to examine Stein's (1966) conditioned inhibitory response theory of habituation as opposed to Sokolov's (1963) neuronal model theory (Ely, 1972; Öhman, 1973). In the latter experiments, for example, participants heard an innocuous tone of constant duration presented with varying ISIs during training. Generalization of habituation tests were introduced by increasing or decreasing the duration of the tone presented under different ISIs. Stein's (1966) theory of habituation suggests that onset of a stimulus evokes an excitatory process as an unconditioned response and as the result of conditioning increasingly evokes an opposing inhibitory process. Habituation occurs because of the growth and overshadowing of the excitatory process by the inhibitory process. Since these opposed excitatory and inhibitory processes are induced by the onset of a physical stimulus, Stein's theory, as in the case of another influential theory of habituation (Groves \& Thompson, 1970), cannot predict generalization of habituation of measures of the OR as a consequence of changes in temporal relations between stimuli. Regardless of its possible shortcomings, a strength of a neuronal model theory is that it implies that systematic changes in indices of the OR will occur with changes in the pattern of the temporal relations of a stimulus as well as with changes in the physical parameters of that stimulus.

It must be further noted that, in the temporal generalization paradigm used by Ely (1972) and Öhman (1973), a number of important variables are confounded in the experimental manipulations. The critical significant stimulus, the tone, is also the stimulus that is changed-is the source of novelty. In changing the duration of the tone, for example shortening it, there is also a change in stimulus energy and possibly arousal as well. Temporal generalization in the present experiment does not involve a change in 
stimulus energy. Novelty and significance are also independent in that novelty involves changes in the temporal interval between occasions of the stimulus which itself does not change. Significance is varied by imparting the tone with signal value for an overt response in one group of students but not another.

\section{METHOD}

Subjects

A total of 112 undergraduate students, 56 men and 56 women, participated in the experiment as a means of fulfilling a course requirement. There were an equal number of men and women within each subgroup.

\section{Procedure}

The experiment was conducted in a two-room suite with a oneway observation mirror between the two rooms. All of the recording and stimulus-programming equipment was in the observation room. The student's room was electrically shielded and semisoundproofed. It contained a comfortable padded chair with foam-rubber armrests. One channel of a stereophonic tape deck was programmed to activate the amplified signals of a frequency generator transmitted to the student via stereophonic headphones at the appropriate temporal intervals. The electrodes were explained to the student, and he or she was told that the experimenters were interested in studying the physiological correlates of relaxation. The student was also told that he or she would hear occasional tones. Each student in the experimental group was instructed to lift his or her foot as fast as possible from the pedal on which it was resting whenever he or she heard the tone.

The stimulus was a .5-sec-duration $1,000-\mathrm{Hz}$ tone with an intensity of approximately $70 \mathrm{~dB}(\mathrm{~A})$ calibrated at the earphones. Sixteen tones with an ISI of $12 \mathrm{sec}$ made up the initial training phase of the experiment. Interstimulus intervals constituting a generalization test were $5.5,7.0,12.0,15.5,20.0$, and $26.0 \mathrm{sec}$, respectively. These intervals are separated by approximately equal $\log$ units. Interstimulus generalization test intervals were presented in seven different orders to seven different subgroups in a nonbalanced Latin square. Three additional training and test series followed the first training series of 16 tone presentations and its generalization test. Each additional series consisted of four training trials with the original 12-sec ISI followed by a generalization test. Each generalization test was administered according to a different randomly selected $7 \times 7$ Latin square. Successive training and test series followed each other without interruption.

\section{Apparatus}

Bipolar palmar GSRs were recorded using two 20-mm disk electrodes fitted in 5-mm-deep plastic cups containing electrode paste made from agar and a $1 \%$ zinc sulfate solution. Plastic pressure clips encased in foam rubber held the electrodes in place. Palmar resistance was continuously recorded using a 40- $\mu \mathrm{A}$ Wheatstone bridge and one channel of a dual-channel Sanborn $320 \mathrm{dc}$ amplifierrecorder. An event marker recorded the occurrence of the stimuli on the polygraph record. Paper speed was $1 \mathrm{~mm} / \mathrm{sec}$. Reaction times (RTs) were obtained by having each student in the experimental group lift his or her foot from a Stenorette pedal especially adapted for this task as soon as he or she heard the tone. The RTs were measured in milliseconds by an electronic counter.

\section{Dependent Variables}

The GSR was defined as the largest decrease in palmar resistance commencing .5-5 sec after stimulus onset. Raw scores were subjected to a log conductance change transformation prior to statistical treatment. Pedal release performance was transformed to a speed measure defined as $1 / R T \times 100$. It was available, of course, only for the experimental students instructed to perform an overt pedal response to the tone signal. The .05 level of signifi- cance for a two-tailed test was adopted in all statistical tests conducted unless otherwise noted.

\section{RESULTS}

Limitations in our computer programs necessitated the use of two types of analyses of variance and orthogonal polynomials in our examination of each set of data. Variables examined in the first analyses were the order of the intervals within each generalization test, sex, instructions or groups, trials, generalization interval, and Latin square in the four different test series, when applicable. In the second analyses, in order to examine the effect of individual differences in OR level, the seven subgroups receiving the different orders of test intervals were collapsed and participants were categorized into high and low OR subgroups within each sex within the experimental and the control groups. Levels were established on the basis of the magnitude of the GSR to the first tone presented at the start of training.

\section{Performance}

Analyses of speed of responding to the first training series of 16 tones presented with a constant 12 -sec ISI yielded significant effects for sex $[F(1,42)=7.88$, $\mathrm{MSe}=32,382.563]$ and trials $[\mathrm{F}(15,630)=24.37, \mathrm{MSe}=$ $11,004.449]$. Mean speed for men was .47 , and for women, .36. Practice effects appeared to reach asymptote by the seventh trial. Individual differences in the level of the initial GSR reflecting an OR to the novelty of the first stimulus was not significantly related to the speed of responding.

Figure 1 shows the mean speed of responding to the tone following various ISIs, the generalization gradient for speed following training at an ISI of $12 \mathrm{sec}$. Results are presented for the first series of test trials. It is apparent that response speeds were slower at intervals shorter and longer than the training interval. Analyses of variance yielded significant effects for ISI $[\mathrm{F}(6,252)=3.17, \mathrm{MSe}=8,599.622]$ and $\operatorname{sex}[F(1,42)=8.08, M S e=118,854.322]$, with men

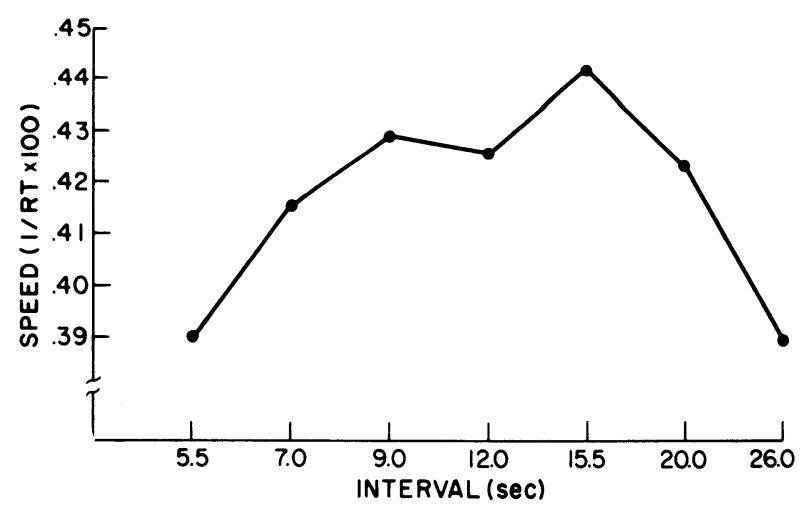

Figure 1. Temporal generalization of mean response speed on the first generalization test series. 
again manifesting greater speed than women. Analyses of the mean speed obtained on all four generalization test trials yielded similar significant effects. None of the sources of variance related to individual differences in the GSR index of the initial OR were significant on the first generalization test series or all four series of generalization tests.

\section{GSR}

Figure 2 illustrates the mean GSR evoked by the 16 tone presentations at a constant ISI of $12 \mathrm{sec}$ during the training or habituation phase. Results are plotted for the high $(\mathrm{H})$ and low (L) OR subgroups within the experimental (E) and control (C) groups. It is apparent that the task provided the experimental group had a significant effect upon GSR responsivity $[\mathrm{F}(1,56)=63.39$, $\mathrm{MSe}=6.366]$. It is apparent also that high and low OR groups differed significantly $[\mathrm{F}(2,56)=14.50, \mathrm{MSe}=6.366]$. Habituation is reflected by the significant effect for trials $[\mathrm{F}(15,840)=54.48, \mathrm{MSe}=.241]$. Decrease in responsivity occurred at different rates for the two groups, as indicated by the significant groups $\times$ trials interaction $[\mathrm{F}(15,840)=4.92, \mathrm{MSe}=.241]$. Analyses of orthogonal polynomials provide additional evidence of significant trend effects. There was a significant overall linear component to the decline in GSR responsivity $[F(1,56)=214.23]$, a significant difference in the linear components of the trends evidenced by the high and low OR groups $[F(1,56)=7.24]$, and an interaction in the groups $\times$ OR linear trend components $[\mathrm{F}(1,56)=5.87, \mathrm{MSe}=.045]$. These interactions in the trends of high and low OR students and the experimental and control groups are evident in Figure 2.

There was also a significant overall quadratic component to the habituation trends $[F(1,56)=84.56]$

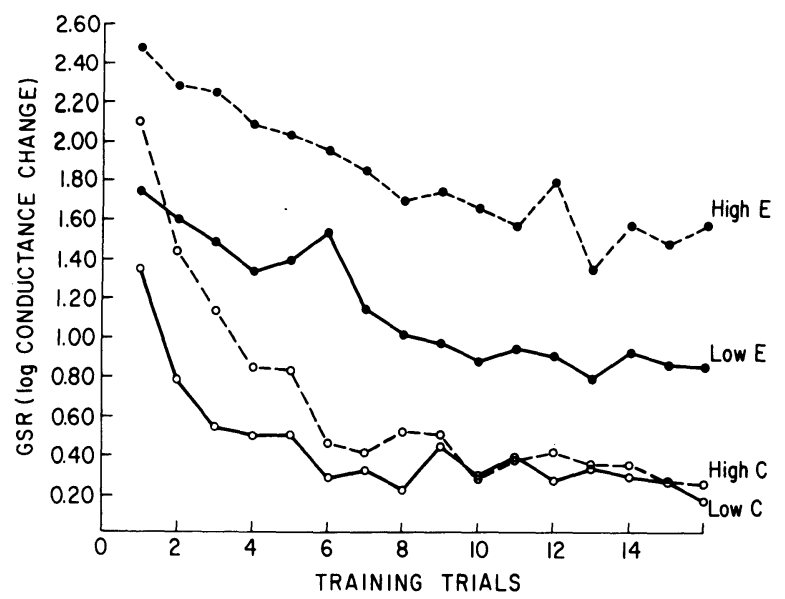

Figure 2. Mean GSR evoked by tones for high (H) and low (L) OR students in the experimental (E) and control (C) groups during the habituation phase with constant 12-sec ISI.

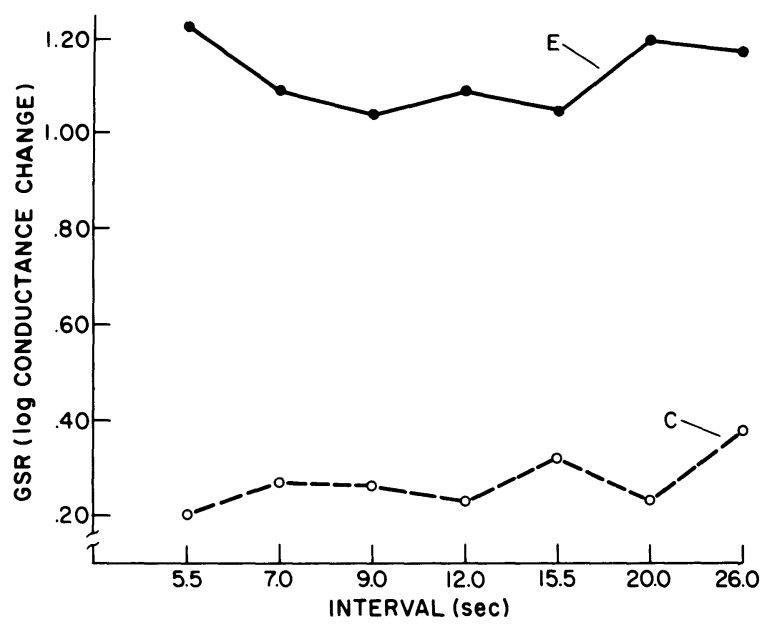

Figure 3. Temporal generalization of the mean GSR on the first test series for the experimental (E) and control (C) groups.

and significant differences in the quadratic components of the trends for groups $[F(1,56)=12.22]$ and level of OR $[\mathrm{F}(1,56)=4.36, \mathrm{MSe}=.023]$.

Results of the first temporal generalization test are shown in Figure 3. It depicts the mean GSRs induced following the different ISIs constituting the first generalization test series for the experimental and control groups. Most apparent is the overall greater level of responsivity displayed by the experimental as compared with the control group. There is also the suggestion of a bidirectional generalization gradient in the experimental group, whereas an asymmetrical gradient appears to be present in the control group. Longer, but not shorter, intervals were accompanied by increased GSR responsivity in the latter group.

Analyses of variance and orthogonal polynomials tend to support the above observations. Groups differed significantly in their overall level of responsivity $[\mathrm{F}(1,84)=42.14, \mathrm{MSe}=3.403]$. There was an overall significant quadratic component in the generalization trends for the two groups $[F(1,84)=5.27$, $\mathrm{MSe}=.161]$, indicating that some generalization of the GSR occurred in the two groups. Generalization gradients differed for the two groups, however, as indicated by a significant difference in the cubic component of their curves $[\mathrm{F}(1,84)=4.02, \mathrm{MSe}=.125]$. It reflects the difference between the symmetrical bow-shaped generalization gradient obtained by the experimental group and the asymmetrical gradient produced by the control group.

Individual comparisons of training with test intervals by means of $t$ tests (one-tailed) within the control and experimental conditions were conducted. It was found that only the 26 -sec interval within the control condition produced significantly greater responsivity than the training interval. Within the experimental condition, the difference between the 
5.5-sec test interval and the training interval approached significance. No other test interval differed significantly from the training interval.

Analyses of variance and orthogonal polynomials indicated the presence of significant generalization trends over all four generalization tests, but no one of the test intervals differed significantly from the training interval within the control or experimental conditions.

It was also found that OR level yielded a significant main effect $[\mathrm{F}(1,104)=23.10, \mathrm{MSe}=2.734]$. High OR subgroups overall were more responsive than low OR subgroups. They did not, however, differ significantly in the shape of their generalization gradients, and OR level did not interact with the experimental conditions.

Each of the seven different test ISIs appeared in each of the seven ordinal positions of the generalization test. It was therefore possible to examine order effects within and between generalization tests. As expected (Maltzman \& Langdon, 1969), the order in which the intervals appeared during the first generalization test produced a significant effect $[F(6,336)$ $=2.56, \mathrm{MSe}=.167]$. By and large, the earlier interval changes, regardless of the size of the interval, induced larger GSR-ORs than did later changes. Mean GSRs for the seven ordinal positions were $.76, .78$, $.71, .70, .63$, and .61 , respectively. Ordinal position failed to yield a significant effect over all four generalization test series. Examination of the effects of repetition of the generalization test indicated a significant amount of habituation, as reflected by a main effect for repetitions $[\mathrm{F}(3,168)=6.59, \mathrm{MSe}=$ .314]. Amount of generalization of the GSR decreased-habituated-with repeated generalization tests.

\section{DISCUSSION}

As expected, magnitude of the GSRs evoked by the tones during training and magnitude of the GSRs evoked by the changed ISIs were significantly greater in the experimental group than in the control group. More pertinent to our theoretical interests, however, was the finding that both groups provided some evidence of heightened responsivity to generalization test intervals as compared with the training interval. Some evidence of a generalization gradient of the GSR index of the OR was obtained when temporal novelty was introduced between both innocuous nonsignal tones and imperative signal tones.

Results obtained with the innocuous nonsignal tone are relevant to the current controversy concerning significance as a determiner of the OR (Bernstein, 1979; Maltzman, 1979; O'Gorman, 1979; Siddle, 1979). Highly reliable evidence of GSR responsivity to the innocuous nonsignificant tone and its habituation during training and some evidence of temporal generalization during the generalization test contradict Bernstein's (1979) hypothesis that significance is a necessary condition for the occurrence of an OR.

On the other hand, the difference between the experimental and control groups in the shapes of their GSR-OR generalization gradients suggests that the significance of stimuli may predetermine, to some degree, the effects of novelty. Changes to shorter ISIs between test tones tended to produce augmented GSR-ORs only when the temporal changes occurred between signal stimuli. Change to longer ISIs tended to produce augmented GSRs in the experimental and control conditions. Thus, to some degree, the effects of novelty, or change, the collative variables emphasized by Berlyne (1960), are dependent upon the state of the individual at the moment, sets, motives, attitudes, interests, or prepotent response tendencies, related notions we would subsume under the conception of a dominant focus or cortical set (Maltzman, 1979a, 1979b).

It is also interesting to note that response speed displayed a symmetrical generalization gradient over all four generalization training and test series as well as on the the first generalization test series. Generalization of the GSR in the experimental group tended to show a symmetrical gradient of generalization only on the first test series. There was a dissociation between the generalization gradients for response speed and the GSR during the course of repeated training and generalization test series.

It is possible to interpret the magnitude of differences in the GSR habituation and generalization gradients between the experimental and control groups in terms of differences in arousal (O'Gorman, 1979). We hesitate, however, for at least two reasons. First, there is the continued theoretical and experimental ambiguity associated with the concept of arousal (Lacey, 1967; Maltzman, 1975; Näätänen, 1973) and the absence of an acceptable measure of arousal in the present experiment. Second, results from other experiments have demonstrated the importance of significance as a predeterminer of the GSR index of the OR when differential contributions of arousal were clearly not involved (Maltzman et al., 1977; Maltzman \& Raskin, 1979).

To fall back upon an older terminology (Paschal, 1941; Pillsbury, 1908), the differential magnitude of the GSR to signal and nonsignal stimuli indicates that attention is in part a function of set. Psychophysiologically, we may say that the OR is, in part, a function of a dominant focus (Maltzman, 1979a, 1979b). In either terminology, much remains to be done experimentally and theoretically before a truly adequate psychophysiological account of orienting to significant and nonsignificant stimuli can be offered. 


\section{REFERENCES}

BARRY, R. J. The effect of "significance" upon indices of Sokolov's orienting response: A new conceptualization to replace the OR. Physiological Psychology, 1977, 5, 209-214.

BeCker, D. E., \& Shapiro, D. Directing attention toward stimuli affects the P300 but not the orienting response. Psychophysiology, 1980, 17, 385-389.

Berlyne, D. E. Conflict, arousal and curiosity. New York: McGraw-Hill, 1960.

Bernstein, A. S. The orienting reflex as novelty and significance detector: Reply to O'Gorman. Psychophysiology, 1979, 16, 263-273.

Desiderato, 0 . Effect of anxiety and stress on reaction time and temporal generalization. Psychological Reports, 1964, 14, 51-58.

ELY, D. J. Temporal duration as a dimension in generalization of the orienting response. Perceptual and Motor Skills, 1972, 34, 271-276.

Groves, P. M., \& Thompson, R. F. Habituation: A dual-process theory. Psychological Review, 1970, 77, 419-450.

LACEY, J. I. Somatic response patterning and stress: Some revisions of activation theory. In M. H. Appley \& R. Trumball (Eds.), Psychological stress: Issues in research. New York: Appleton-Century-Crofts, 1967.

LAtASH, L. P. Hypothalamus, adaptive activity, and the electroencephalogram. Moscow: Nauka, 1968.

LURIA, A. R. The working brain. New York: Basic Books, 1973.

Luria, A. R., \& HomskayA, E. D. Frontal lobes and the regulation of arousal processes. In D. I. Mostofsky (Eds.), Attention: Contemporary theory and analysis. New York: AppletonCentury-Crofts, 1970.

Luria, A. R., \& Vinogradova, O. S. An objective investigation of the dynamics of semantic systems. British Journal of Psychology, 1959, 50, 89-105.

Maltzman, I. The orienting reflex and thinking as determiners of conditioning and generalization to words. In $\mathrm{H}$. $\mathrm{H}$. Kendler \& J. T. Spence (Eds.), Essays in neobehaviorism: A memorial volume to Kenneth $W$. Spence. New York: Appleton-CenturyCrofts, 1971.

Maltzman, I. Comments on conditioning and psychopathology. In M. O. Kietzman, S. Sutton, \& J. Zubin (Eds.), Experimental approaches to psychopathology. New York: Academic Press, 1975.

Maltzman, I. Orienting reflexes and significance: A reply to O'Gorman. Psychophysiology, 1979, 16, 274-282. (a)

Maltzman, I. Orienting reflexes and classical conditioning in humans. In H. D. Kimmel, E. H. van Olst, \& J. F. Orlebeke
(Eds.), The orienting reflex in humans. New York: Erlbaum, 1979. (b)

Maltzman, I., Gould, J., Barnett, O. J., Raskin, D. C., \& WolfF, C. Habituation of the GSR and digital vasomotor components of the orienting reflex as a consequence of task instructions and sex differences. Physiological Psychology, 1979, 7, 213-220.

Maltzman, I., Gould, J., Pendery, M., \& Wolff, C. Semantic conditioning and generalization of the GSR orienting reflex with overt and covert activity. Journal of Experimental Psychology: General, 1977, 106, 172-184.

Maltzman, I., Harris, L., Ingram, E., \& Wolff, C. A primacy effect in the orienting reflex to stimulus change. Journal of Experimental Psychology, 1971, 87, 202-206.

Maltzman, I., \& Langdon, B. Semantic generalization of the GSR as a function of semantic distance for the orienting reflex. Journal of Experimental Psychology, 1969, 80, 289-294.

Maltzman, I., \& Raskin, D. C. Effects of individual differences in the orienting reflex on conditioning and complex processes. Journal of Experimental Research in Personality, 1965, 1, 1-16.

Maltzman, I., \& RAskin, D. C. Selective orienting and habituation of the GSR as a consequence of overt and covert activity. Physiological Psychology, 1979, 7, 204-208.

NÄÄtÄNEN, $R$. The inverted-U relationship between activation and performance: $A$ critical review. In S. Kornblum (Ed.), Attention and performance IV. New York: Academic Press, 1973.

O'Gorman, J. G. The orienting reflex: Novelty or significance detector? Psychophysiology, 1979, 16, 253-262.

ÖHman, A. Temporal generalization of electrodermal orienting responses: Comment. Perceptual and Motor Skills, 1973, 37, 593-594.

Paschal, F. C. The trend in theories of attention. Psychological Review, 1941, 48, 383-403.

Pillsbury, W. B. Attention. New York: Macmillan, 1908.

SiddeE, D. A. T. The orienting response and stimulus significance: Some comments. Biological Psychology, 1979, 8, 303-309.

Sokolov, E. N. Perception and the conditioned reflex. New York: Macmillan, 1963.

STEIN, L. Habituation and stimulus novelty: A model based on classical conditioning. Psychological Review, 1966, 73, 352-356.

(Manuscript received December 5, 1980; revision accepted for publication May 14, 1982.) 